\title{
INFLUÊNCIA DA ESTERILIZAÇÃO SOBRE A CAPACIDADE DE CORTE DOS INSTRUMENTOS ENDODÔNTICOS ROTATÓRIOS PROTAPER ${ }^{\circledR}$
}

\section{INFLUENCE OF THE STERILIZATION ON THE CUT CAPACITY OF ROTATORY ENDODONTICS INSTRUMENTS PROTAPER ${ }^{\circledR}$}

\author{
Graziele Borin* \\ Alex Niederauer Becker* \\ Elias Pandonor Motcy de Oliveira** \\ Tiago André Fontoura de Melo**** \\ Simone Soares Echeveste ${ }^{* * * * *}$
}

\begin{abstract}
RESUMO
Introdução: O propósito deste estudo é analisar o efeito do processo de esterilização sobre a capacidade de corte dos instrumentos endodônticos rotatórios ProTaper. Métodos: Para isso foram utilizados 30 canais simulados e 6 caixas do sistema ProTaper totalizando 36 instrumentos (SX, S1, S2, F1, F2 e F3), seis de cada calibre. Estes foram divididos em dois grupos experimentais: Grupo A: 3 caixas do sistema ProTaper cujo instrumentos foram submetidos ao processo de limpeza e esterilização; Grupo B: 3 caixas de instrumentos que foram submetidos somente ao processo de limpeza. Os canais simulados foram identificados e pesados em balança analítica antes e após a realizaçáo do preparo. Cada caixa de instrumentos foi utilizada em 5 canais simulados, sendo que cada instrumento permaneceu atuando por 10 segundos no seu interior, empregando-se $2 \mathrm{ml}$ de detergente aniônico Tergensol (Inodon) entre cada troca de instrumento. Foi utilizado o teste t-Student para comparação entre os dois grupos experimentais. Resultados e conclusão: Os resultados mostraram que os instrumentos do Grupo B apresentaram uma maior eficiência de corte em relação aos instrumentos do Grupo $\mathrm{A}$, no entanto não houve diferença estatística significativa entre os mesmos $(\mathrm{p}=0,32)$.
\end{abstract}

DESCRITORES: Endodontia - Instrumentos odontológicos - Esterilização -Níquel -Titânio

\begin{abstract} A, however did not have difference significant statistics between the same ones $(\mathrm{p}=0,32)$.

DESCRIPTORS: Endodontics - Dental instruments - Sterilization - Nickel - Titanium

\footnotetext{
* Mestre em Endodontia - Universidade Luterana do Brasil (ULBRA) Canoas/RS.

** Doutor em Endodontia - Professor de Endodontia da Universidade Luterana do Brasil (ULBRA) Canoas/RS.

*** Mestrando em Endodontia - Universidade Luterana do Brasil (ULBRA) Canoas/RS.

**** Mestre em Marketing - Professora de Matemática da Universidade Luterana do Brasil (ULBRA) Canoas/RS.
}

Introduction: The aim of this study is to analyze the effect of the sterilization process on the cut capacity of rotatory endodontics instruments ProTaper'. Methods: For this 30 simulated canals and 6 boxes of the ProTaper ${ }^{\circ}$ system had been used totalizing 36 instruments (SX, S1, S2, F1, F2 and F3), six of each bore. These had been divided in two experimental groups: Group A: 3 boxes of the ProTaper system whose instruments had been submitted to the process of cleanness and sterilization; Group B: 3 boxes of instruments that had been submitted only to the cleanness process. The simulated canals had been identified and weighed in analytical scale before and after the accomplishment of the preparation. Each box of instruments was used in 5 simulated canals, being that each instrument remained acting per 10 seconds in side, using itself $2 \mathrm{ml}$ of anionic detergent Tergensol (Inodon) enters each exchange of instrument. For comparison between the two experimental groups was used t-Student test. Results e Conclusion: The results had shown that the instruments of Group B had presented a bigger efficiency of cut in relation to the instruments of the Group 
Borin G, Becker AN, Oliveira EPM, Melo TAF, Echeveste SS. Influência da esterilização sobre a capacidade de corte dos instrumentos endodônticos rotatórios protaper. Revista de Odontologia da Universidade Cidade de São Paulo 2008 jan-abr; 20(1):14-18

\section{INTRODUÇÃO E REVISÃO DA LITERATURA}

A partir da introdução dos instrumentos endodônticos fabricados com a liga de níquel-titânio por Walia et al. ${ }^{12}$ (1988), a realização do preparo de canais radiculares curvos se tornou muito mais facilitada. Desde entáo, houve o surgimento de inúmeros instrumentos com diferentes desenhos, formas e conicidades sendo que um dos mais utilizados atualmente, devido às suas propriedades, é o ProTaper (Dentsply/ Maillefer, Ballaigues, Switzerland).

Segundo Lopes et al. ${ }^{3}$ (2004) a conicidade variada dos instrumentos ProTaper tem como objetivo combinar a técnica de preparo coroa-ápice e a manutenção da patência do canal radicular com uma seqüência reduzida e simples de instrumentos a serem utilizados. A grande característica dos ProTaper é a sua capacidade de corte; isso se deve ao ângulo agudo da aresta lateral de corte.

Os instrumentos endodônticos são reproduzidos por usinagem e geralmente apresentam defeitos advindos do processo de fabricação, observados na parte ativa do instrumento na forma de ranhuras, microcavidades e rebarbas (Serene et al. ${ }^{8}$ 1995). A presença de rebarbas altera o ângulo e a agudicidade da aresta de corte, diminuindo a capacidade de corte dos instrumentos endodônticos (Lopes et al. ${ }^{3}, 2004$ ).

Além disso, outro fator que pode influenciar na capacidade de corte dos instrumentos endodônticos é a esterilização. Butti $e t ~ a l^{2}{ }^{2}$ (1995) verificaram que após a esterilização de instrumentos endodônticos de aço inoxidável houve uma diminuição nas suas propriedades de corte e essa alteraçâo foi proporcional ao aumento do número de ciclos de esterilização.

Da mesma forma, Shabaloyskaya e Anderegg' (1995) examinaram as superfícies de materiais de níquel-titânio submetidas a inúmeros ciclos de esterilização. A autoclavagem em $120^{\circ} \mathrm{C}$ e 21 psi produziu uma alteração nas concentrações de níquel, titânio, oxigênio e carbono na superfície do material. A extensão da mudança foi proporcional ao tempo do tratamento. Um decréscimo na concentração de níquel foi também encontrado na superfície dos instrumentos com o aumento do tempo de exposição (1-2 horas de autoclavagem).

Rapisarda et al. ${ }^{7}$ (1999) verificaram as alteraçōes na capacidade de corte de instrumentos de níquel-titânio quando submetidos a 7 e 14 ciclos de autoclavagem, comparando-os com um grupo-controle (não esterilizado). Os instrumentos que sofreram 7 ciclos de esterilização perderam $20 \%$ da sua capacidade de corte e os que foram submetidos a 14 ciclos tiveram sua capacidade de corte reduzida para menos de $50 \%$. Os autores atribuíram essa perda de capacidade de corte à deposição de uma camada de oxigênio na superfície do instrumento após sua esterilização em autoclave, verificada através da Espectroscopia Auger.

Com a diminuição da capacidade de corte dos instrumentos de níquel-titânio, a dificuldade de corte da dentina induz o operador a aumentar o carregamento imposto ao instrumento durante o preparo do canal radicular o que pode levar à fratura do instrumento (Lopes et $\left.a l .^{3}, 2004\right)$. A fratura dos instrumentos de níquel-titânio ocorre com razoável freqüência e de modo inesperado, sem que apresentem sinal prévio de deformação, o que leva muitos profissionais a abandonarem seu uso.

Diante disso, o propósito deste estudo é analisar o efeito do processo de esterilizaçáo sobre a capacidade de corte dos instrumentos endodônticos rotatórios do sistema ProTaper.

\section{MATERIAIS E MÉTODOS}

Para a realização da parte experimental, foram utilizados 30 canais simulados (Odontofix - Ribeirão Preto/ SP) com diâmetro inicial igual ao de um instrumento endodôntico de aço inoxidável número 20, apresentan-

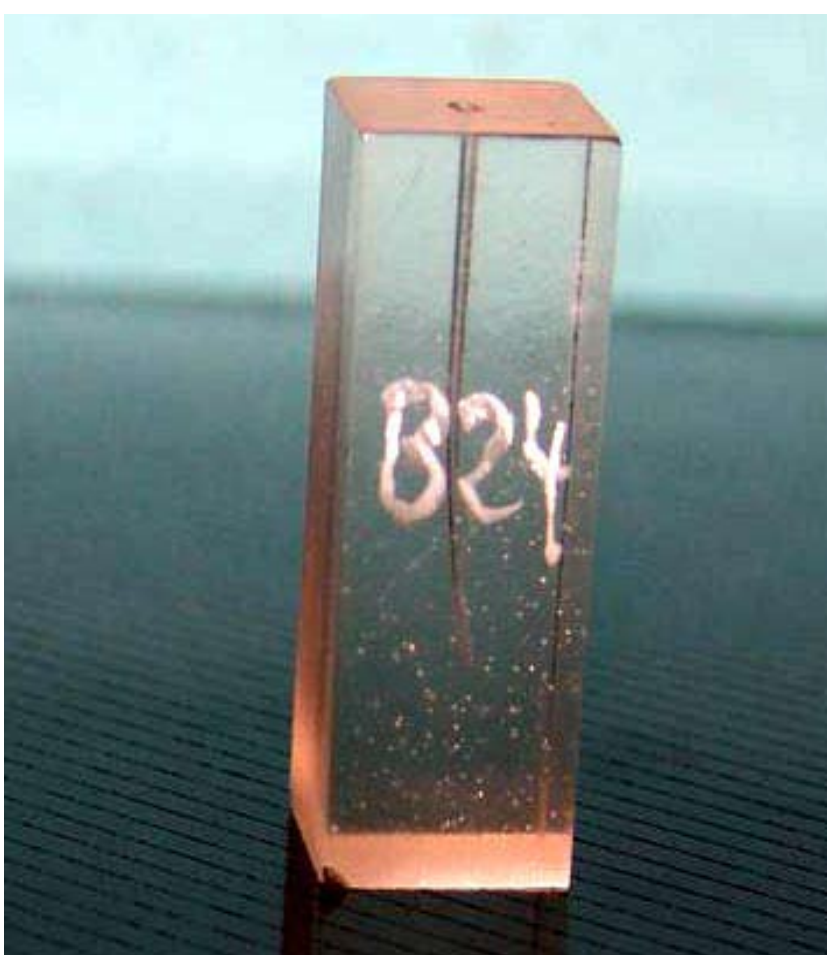

Figura 1 - Canal simulado utilizado na parte experimental. 
Borin G, Becker AN, Oliveira EPM, Melo TAF, Echeveste SS. Influência da esterilização sobre a capacidade de corte dos instrumentos endodônticos rotatórios protaper. Revista de Odontologia da Universidade Cidade de São Paulo 2008 jan-abr; 20(1):14-18

do 18 milímetros de comprimento, cuja curvatura começava a 10 milímetros da extremidade final do canal, tendo seu maior grau a 5 milímetros, apresentando nesta regiâo uma curvatura de 20 graus (Figura 1).

Esses canais foram devidamente numerados de 01 a 30 e pesados em balança analítica de alta precisão (Adventurer ${ }^{\mathrm{TM}}$ Ohaus).

Utilizaram-se também, 36 instrumentos endodônticos rotatórios ProTaper(Dentsply/ Maillefer) perfazendo 6 caixas, cada uma composta pelos instrumentos $S_{X}, S_{1}$, $\mathrm{S}_{2}, \mathrm{~F}_{1}, \mathrm{~F}_{2}$ e $\mathrm{F}_{3}$ (Figura 2)

Os instrumentos foram divididos em dois grupos ex-

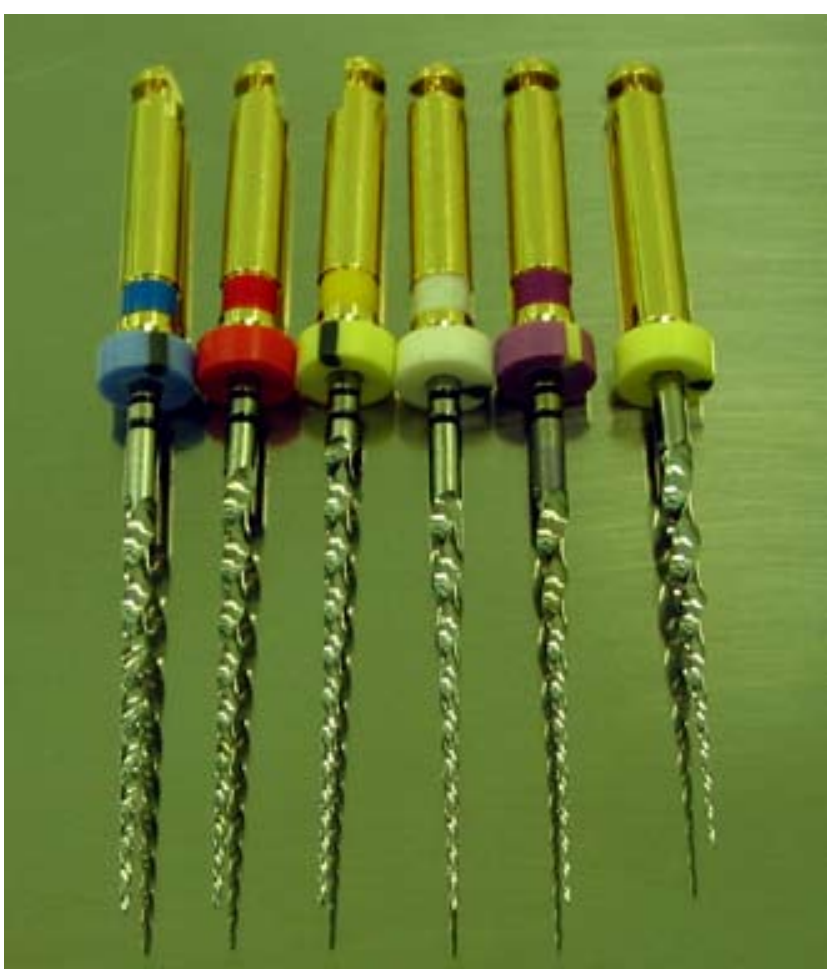

Figura 2 - Instrumentos endodônticos rotatórios ProTaper ${ }^{\odot}$ utilizados no experimento.

perimentais (Quadro 1):

GRUPO A: 3 caixas do sistema ProTaper, cujos instrumentos foram submetidos ao processo de limpeza e esterilização em autoclave;

GRUPO B: 3 caixas do sistema ProTaper, cujos instrumentos foram submetidos somente ao processo de limpeza sem passar pela esterilização.

O processo de limpeza foi realizado logo após a remoção dos instrumentos endodônticos da caixa e após o preparo dos canais simulados. Seguiu-se a técnica preconizada por Queiróz ${ }^{6}$ (2001), que realizou a ultra-
Quadro 1 - Quadro demonstrativo dos grupos experimentais.

\begin{tabular}{ccccc}
\hline \hline Grupo & $\begin{array}{c}\mathrm{N}^{\circ} . \\
\text { Canais }\end{array}$ & $\begin{array}{c}\mathrm{N}^{\circ} . \\
\text { Caixas }\end{array}$ & $\begin{array}{c}\text { Tratamento } \\
\text { aplicado nos } \\
\text { instrumento }\end{array}$ & $\begin{array}{c}\text { No. Ciclos } \\
\text { de Esteri- } \\
\text { lização }\end{array}$ \\
\hline A & 15 & 3 & $\begin{array}{c}\text { Limpeza }+ \\
\text { Esterilização }\end{array}$ & 6 \\
B & 15 & 3 & Limpeza & - \\
\hline \hline
\end{tabular}

sonificação dos instrumentos em uma cuba ultra-sônica contendo detergente enzimático Riozyme II (Rioquímica, São Paulo/SP) por um tempo de 20 minutos. Em seguida, procedeu-se à escovação com escova dental de cerdas macias (Oral-B, São Paulo/SP) umedecidas com sabão líquido, lavagem em água corrente e secagem em estufa a $50^{\circ} \mathrm{C}$ por 10 minutos.

Os instrumentos do Grupo A foram embalados em envelopes próprios para esterilização em autoclave (Rexam, São Paulo/SP), e foram submetidos a esterilização logo após a remoção da caixa, ou seja, antes do primeiro uso e após cada um dos cinco usos, totalizando 6 ciclos. Cada ciclo consistia na exposição ao calor úmido, a 1 ATM de pressão e $127^{\circ} \mathrm{C}$, por vinte minutos (Brasil. Ministério da Saúde ${ }^{1}$, 2000).

Para o preparo dos canais simulados, utilizou-se o motor Endo pro torque (VK Driller Equipamentos Elétricos Ltda, São Paulo/SP) numa velocidade de $250 \mathrm{rpm}$ e torque de $2 \mathrm{~N}$. Todos os instrumentos foram empregados de acordo com a cinemática indicada pelo fabricante. Cada caixa de instrumentos foi empregado em 5 canais simulados, e cada instrumento permaneceu atuando por 10 segundos no seu interior, realizando-se irrigação com $2 \mathrm{ml}$ de detergente aniônico Tergensol (Inodon - Porto Alegre/RS) e aspiração entre cada troca de instrumento. Os canais foram preparados sempre pelo mesmo operador, que não conhecia os grupos experimentais.

Após a realizaçáo do preparo, os canais simulados foram irrigados com detergente aniônico, aspirados e secos à temperatura ambiente para que se pudesse realizar a pesagem final (após o preparo dos canais), tomando-se o cuidado para utilizar a mesma balança analítica utilizada na pesagem inicial (antes do preparo dos canais). Com os valores obtidos na pesagem, foi possível verificar a eficiência de corte dos instrumentos através da comparação da média do desgaste dos grupos A e B. Para a análise dos resultados foi utilizado o teste $\mathrm{t}$-Student $(\mathrm{p}=0,32)$. 
Borin G, Becker AN, Oliveira EPM, Melo TAF, Echeveste SS. Influência da esterilização sobre a capacidade de corte dos instrumentos endodônticos rotatórios protaper. Revista de Odontologia da Universidade Cidade de São Paulo 2008 jan-abr; 20(1):14-18

\section{RESULTADOS}

$\mathrm{Na}$ comparação da eficiência de corte entre os dois grupos experimentais (Gráfico 1) e entre o número de usos dos instrumentos endodônticos (Gráfico 2), o Grupo $B$ apresentou maior eficiência de corte em relação ao Grupo A, embora não tenha ocorrido diferença estatisticamente significante ao nível de 5\%.

Gráfico 1 - Gráfico representando a eficiência de corte de acordo com os grupos experimentais.

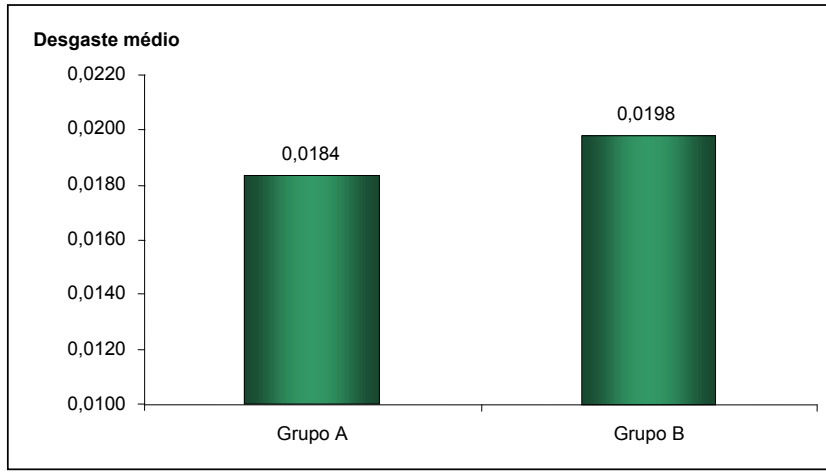

Gráfico 2 - Gráfico representando a eficiência de corte de acordo com o número de usos.

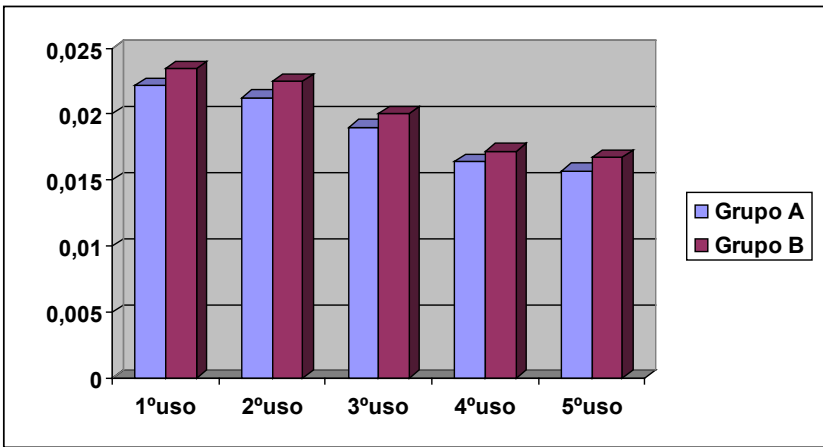

\section{DISCUSSÃO}

A utilização, cada vez mais freqüente, de instrumentos endodônticos rotatórios de níquel-titânio pelos endodontistas, tem aumentado o interesse dos pesquisadores em se obterem melhores resultados com esse tipo de instrumento. Um dos fatores mais importantes que pode influenciar o uso clínico diário desses instrumentos é o risco de sua fratura.

Conforme Lopes et al. ${ }^{3}$ (2004), a diminuição da capacidade de corte do instrumento endodôntico de níquel-titânio pode induzir o operador a aumentar o carregamento, podendo ocasionar a sua fratura. Está comprovado que a esterilização em autoclave promove alterações nas concentrações de níquel e de titânio e uma diminuição nas propriedades de corte desses instrumentos (Shabalovskaya e Anderegg ${ }^{9}$, 1995; Rapisarda et al. ${ }^{7}$, 1999).

Diante disso, nosso estudo se propóe a verificar se a esterilização em autoclave promove alteraçóes na capacidade de corte dos instrumentos endodônticos rotatórios ProTaper. Optou-se por estes instrumentos em função de sua ampla utilização e por apresentarem excelentes resultados clínicos. Buscando-se uma padronizaçáo com relação à curvatura e diâmetro dos canais radiculares, utilizaram-se canais simulados que, segundo Weine et al. ${ }^{13}$ (1976); Patterson ${ }^{4}$ (1963); Troian et al. ${ }^{10}$ (2006), apresentam dureza similar à dentina humana.

Quanto aos resultados encontrados, pode-se verificar que os instrumentos endodônticos utilizados no grupo B (não esterilizados) apresentaram maior eficiência de corte em relação aos instrumentos do Grupo A (esterilizados) embora não tenha ocorrido diferença estatística significativa. Levando-se em consideração que a diferença do desgaste médio dos canais entre os dois grupos foi de $0,0014 \mathrm{mg}$, pode-se concluir que a esterilização não interferiu na capacidade de corte dos instrumentos endodônticos analisados.

Em relação ao número de usos, verificou-se que os instrumentos endodônticos do Grupo B apresentaram melhor eficiência de corte em todos os usos, em relação aos instrumentos endodônticos do Grupo $\mathrm{A}$, porém não houve diferença estatística significante ao nível de 5\%. Em ambos os grupos houve um decréscimo da eficiência de corte com o decorrer dos usos.

Cabe ressaltar que, neste estudo, os instrumentos foram utilizados em apenas cinco canais simulados, assim como relatam os trabalhos de Pessoa ${ }^{5}$ (2003); Yared e Kulkarni ${ }^{14}$ (2003); Veltri et al. ${ }^{11}$ (2004); Troian et al. ${ }^{10}$ (2006). Assim, foram submetidos a apenas 6 ciclos de esterilizaçóes. Diferentemente do trabalho de Rapisarda et al..$^{7}$ (1999), que verificou uma diminuição de $20 \%$ na capacidade de corte dos instrumentos quando submetidos a 7 ciclos de esterilizaçóes e de $50 \%$ quando submetidos a 14 ciclos.

\section{CONCLUSÃO}

A capacidade de corte dos instrumentos endodônticos rotatórios ProTaper não foi afetada após o emprego de 6 ciclos de esterilização em autoclave ao nível estatístico de $5 \%$. 


\section{REFERÊNCIAS}

1. Brasil. Ministério da Saúde. Secretaria de Políticas de Saúde, Coordenação Nacional de DST e Aids. Controle de infecções e a prática odontológica em tempos de AIDS; manual de condutas. Brasília: Ministério da Saúde; 2000.

2. Butti A, Ferraroni M, RE D. Influenza delle techiche di sterilizazione rapida sulle proprietà meccaniche degli strumenti endodontici. G Ital Endod. 1995;9:144-50.

3. Lopes HP, Siqueira Jr JFS, Elias CN. Mecanismo de fratura dos instrumentos endodônticos. In: Lopes HP, Siqueira Jr JFS. Endodontia biologia e técnica. 2 ed. Rio de janeiro: Guanabara Koogan; 2004. p.481.

4. Patterson SS. In vivo and in vitro studies of the effect of the disodium slat of ethylenediamine tetra-acetate on human dentine and its endodontic implications. Oral Surg Oral Med Oral Pathol 1963 Jan; 16:83-103.

5. Pessoa OF. Resistência à fadiga cíclica de instrumentos rotatórios de níquel-titânio em razão do uso. [Doutorado] São Paulo: Faculdade de Odontologia da Universidade de São Paulo; 2003.

6. Queiróz MLP. Avaliação comparativa da eficácia de diferentes técnicas empregadas na limpeza de limas endodônticas. [Mestrado] Canoas: Faculdade de Odontologia da Universidade Luterana do Brasil; 2001.

7. Rapisarda E, Bonaccorso A. Tripi TR, Condorelli GG. Effect of sterilization on the cutting efficiency of rotary nickel-titanium endodontic files. Oral Surg Oral Med Oral Pathol Oral Radiol Endod.1999 Sep; 88(3):343-7.
8. Serene TP, Adams JD, Saxena A. Nickel-titanium Instruments: applications in endodontics. St. Louis, Missouri, USA: Ishiyaku Euroamerica; 1995.

9. Shabalovskaya SA, Anderegg JW. Surface spectroscopic characterization of NiTi nearly equitamic shape memory alloys for implant. J Vac Sci Technol, 1995; 13:2624-32.

10. Troian $\mathrm{CH}$, Só MV, Figueiredo JA, Oliveira EP. Deformation and fracture of $\mathrm{RaCe}$ and $\mathrm{K} 3$ endodontic instruments according to the number of uses. Int EndodJ 2006 Aug; 39(8): 616-25.

11. Veltri M, Mollo A, Pini PP, Gheli LF, Balleri P. In vitro comparison of shaping abilities of ProTaper ad GT rotary files. J Endod 2004 Mar; 30(3):163-6.

12. Walia HM, Brantley WA, Gerstein H. An initial investigation of the bending and torsional properties of Nitinol root canal files. J Endod 1988 Jul; 14(7): 346-51.

13. Weine FS, Kelly RF, Bray KE. Effect of preparation with endodontic handpieces on original canal shape. J Endod 1976 Oct; 2(10):298-303.

14. Yared G, Kulkarni GK. An in vitro study of the torsional properties of new and used rotary nickel-titanium files in plastic blocks. Oral Surg Oral Med Oral Pathol Oral Radiol Endod 2003 Oct; 96(4):466-71

Recebido em: 05/06/07 Aceito em: 12/01/07 